This is the accepted manuscript made available via CHORUS, the article has been published as:

\title{
Zero-differential conductance of two-dimensional electrons in crossed electric and magnetic fields
}

A. A. Bykov, Sean Byrnes, Scott Dietrich, Sergey Vitkalov, I. V. Marchishin, and D. V.

Dmitriev

Phys. Rev. B 87, 081409 - Published 25 February 2013

DOI: 10.1103/PhysRevB.87.081409 


\title{
Zero-differential conductance of two-dimensional electrons in crossed electric and magnetic fields
}

\author{
A. A. Bykov, ${ }^{*}$ Sean Byrnes, ${ }^{\dagger}$ Scott Dietrich, ${ }^{\dagger}$ and Sergey Vitkalov ${ }^{\ddagger}$ \\ Physics Department, City College of the City University of New York, New York 10031, USA \\ I. V. Marchishin and D. V. Dmitriev \\ Rzhanov Institute of Semiconductor Physics, Siberian Branch, \\ Russian Academy of Science, 630090 Novosibirsk, Russia
}

(Dated: February 6, 2013)

\begin{abstract}
An electronic state with zero-differential conductance is found in nonlinear response to an electric field $E$ applied to two dimensional Corbino discs of highly mobile carriers placed in quantizing magnetic fields. The state occurs above a critical electric field $E>E_{t h}$ at low temperatures and is accompanied by an abrupt dip in the differential conductance. The proposed model considers a local instability of the electric field $E$ as the origin of the observed phenomenon. Comparison between the observed electronic state and the state with zero differential resistance, occurring in Hall bar geometry, indicates that the nonlinear response of edge states and/or skipping orbits is not essential in the studied samples. The result confirms that quantal heating is the dominant nonlinear mechanism leading to electronic states with both zero differential resistance and conductance.
\end{abstract}

PACS numbers: 72.20.My, 73.43.Qt, 73.50.Jt, 73.63.Hs

The recent interest in a comprehensive study of the nonlinear magnetotransport in two dimensional (2D) electron systems was stimulated by an observation of the Zener tunneling of highly mobile 2D electrons between Landau levels, which is induced by Hall electric field in GaAs/AlGaAs heterojunctions ${ }^{1}$. The effect was originally found in Hall bar geometry and appeared as oscillations of magnetoresistance $r_{x x}(B)$ induced by dc electric current $I_{d c}$. Positions of the oscillations in magnetic field $\mathrm{B}$ obeyed the following relation: $\gamma R_{c} e E_{H}=l \hbar \omega_{c}$, where $\gamma \approx 2, l$ is an integer, $\omega_{c}$ is the cyclotron frequency, $R_{c}$ is the cyclotron radius and $E_{H}$ is Hall electric field. Later, the Zener oscillations of the magnetoresistance $r_{x x}$ were found in highly doped GaAs quantum wells ${ }^{2}$, in double quantum wells ${ }^{3}$ and in hole gas ${ }^{4}$. Very recently the Zener oscillations are detected in the differential conductance of Corbino discs, where the Hall electric field $E_{H}$ is absent ${ }^{5}$.

Another intriguing nonlinear phenomenon that is observed in $2 \mathrm{D}$ electron systems placed in crossed electric and quantizing magnetic fields, is the electronic state with zero differential resistance(ZDR state) ${ }^{6}$. The experimental data have demonstrated, that in the Hall bar geometry the initial decrease of the longitudinal differential resistance $r_{x x}$ with applied dc current $I_{d c}$ terminates at $I_{d c}=I_{t h}$ corresponding to $r_{x x}=0$. At $I_{d c}>I_{t h}$ the differential resistance stays at zero value in a broad range of electric currents $I_{d c}>I_{t h}$, significantly exceeding the threshold value $I_{t h}$. The initial drop of the resistance is associated with a quantal heating induced by the spectral diffusion of $2 \mathrm{D}$ electrons in crossed electric and magnetic fields ${ }^{7-10}$. The transition into the ZDR state is attributed to the local instability of the electric current at $I_{d c}>I_{t h}{ }^{11}$. The local instability is considered to be the origin of another spectacular phenomenon - the zero resistance state observed in highly mobile $2 \mathrm{D}$ electron systems under a microwave irradiation ${ }^{12-14}$. We note that an uncertainty of the microwave field distribution in studied samples limits the quantitative comparison of the nonlinear response with theories. Presented below data are obtained in low frequency domain, where the distribution of the electric field is considered to be quite well determined.

Recently a strong nonlinear response of two dimensional electrons was observed in a geometry in which a nonlocal electron transport, associated with the propagation of the edge states or/and skipping orbits ${ }^{16-23}$, plays the dominant role ${ }^{15}$. The observation of the nonlocal nonlinear response has raised a question regarding a possibility of the significant contribution of the edge states and/or skipping orbits to the nonlinear transport of $2 \mathrm{D}$ electrons observed in the Hall bar geometry ${ }^{24-35}$ and, thus, the applicability of the currently accepted theoretical approach ${ }^{7}$ to the observed nonlinearity. We should note that in the Hall bar geometry a separation between the local and the nonlocal contributions to the electron conductance is a challenging problem.

A convenient geometry in which the nonlocal contributions of the edge states and/or skipping orbits to the electron conductance can be significantly suppressed is the Corbino geometry. In this geometry the edge states are localized near the edges of the inner and outer contacts and do not propagate through the Corbino ring. Thus experiments in the Corbino geometry provide the information on the bulk nonlinear response. A comparison of the nonlinear response of Corbino discs with the response of Hall bar samples may shed a light on the amount of the nonlocal contributions to the nonlinear resistance in the Hall bar geometry. Below we investigate the nonlinear response of Corbino discs and compare it with experiments on Hall bar samples.

The paper presents a study of nonlinear transport properties of $2 \mathrm{D}$ electron Corbino discs with inner radius 


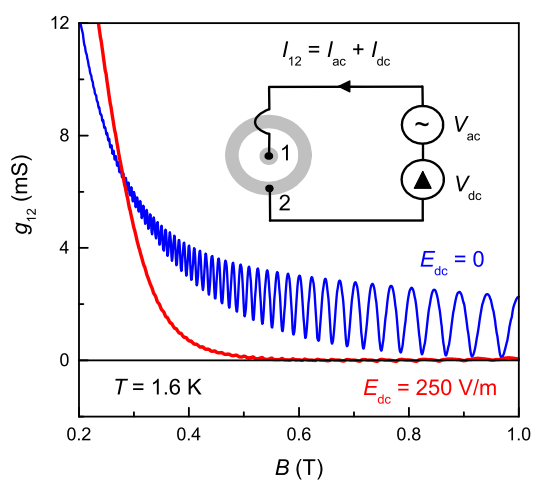

FIG. 1: (Color online.) Dependence of conductance $g_{12}$ of $2 \mathrm{D}$ electron Corbino disc on magnetic field at temperature $T=1.6 \mathrm{~K}$ at different dc electric fields as labeled. Insert shows the electric scheme for measurements of differential conductance $g_{12}$.

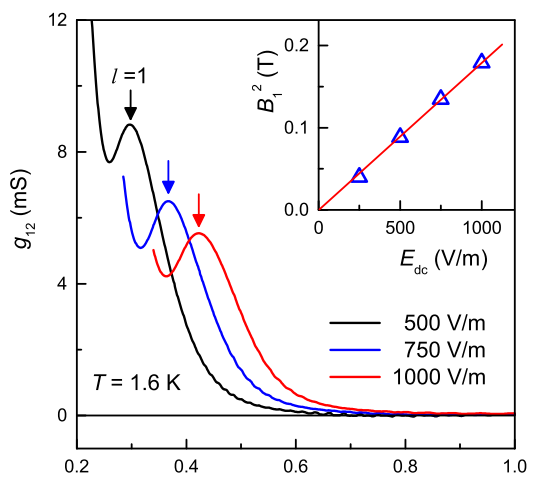

FIG. 2: Dependence of conductance $g_{12}$ of $2 \mathrm{D}$ electron Corbino disc on magnetic field at temperature $T=1.6 \mathrm{~K}$ at different dc electric fields as labeled. Arrows indicate the positions of the maximum $B_{l}$ at $l=1$ in different electric fields. Insert presents the dependence of $B_{1}^{2}$ on dc electric field $E_{d c}$. The solid line corresponds to relation $\gamma e E_{d c} R_{c}=\hbar \omega_{c}$. At $\gamma=2$ the electron effective mass $m_{e} \approx 0.070$, which is in accord with other experiment ${ }^{38}$.

$r_{1}=0.9 \mathrm{~mm}$ and outer radius $r_{2}=1 \mathrm{~mm}$. The Corbino discs were fabricated from selectively doped heterojunction GaAs/AlAs. The heterojunction was a single GaAs quantum well sandwiched between AlAs/GaAs superlattice barriers ${ }^{36}$. The width of the quantum well was 13 $\mathrm{nm}$. The structure was grown by molecular beam epitaxy on (100) GaAs substrate. AuGe eutectic was used to provide electric contacts to the $2 \mathrm{D}$ electron gas. The contacts were made by thermal diffusion after the AuGe deposition and photo-lithography. Differential conductance $g_{12}=I_{a c} / V_{a c}$ were measured using ac current $I_{a c}$ with frequency from $10 \mathrm{~Hz}$ to $1 \mathrm{KHz}$. An ac voltage $V_{a c}$ was applied between contacts 1 and 2 , shown on the insert to Fig.1. The amplitude of the voltage was kept fixed and was below $1 \mathrm{mV}$ during experiments. The measurements were taken at temperatures $\mathrm{T}=1.6 \mathrm{~K}$ and $\mathrm{T}=4.2 \mathrm{~K}$ in magnetic fields $B<1 \mathrm{~T}$. Three samples with electron density $n=8 \cdot 10^{15} \mathrm{~m}^{-2}$ and mobility $\mu=150 \mathrm{~m}^{2} / \mathrm{Vs}$ at $\mathrm{T}=4.2 \mathrm{~K}$ were studied and have demonstrated the same results. The paper presents data for one of these samples.
Figures 1 and 2 present dependence of the differential conductance $g_{12}(B)$ of $2 \mathrm{D}$ electrons in the Corbino disc on the magnetic field $B$ taken at $\mathrm{T}=1.6 \mathrm{~K}$ at different electric fields as labeled. For the studied samples the width of the conducting o-ring was much less than the averaged radius of the o-ring : $\Delta r=r_{2}-r_{1} \ll\left(r_{2}+r_{1}\right) / 2$. Due to this property the dc electric field between contacts was nearly independent of the radius $r$ and equal to $E_{d c}=V_{12} / \Delta r$. At $E_{d c}=0$ the magnetoconductance $g_{12}(B)$ demonstrates Shubnikov de Haas (SdH) oscillations in magnetic fields exceeding $0.3 \mathrm{~T}$ as shown in Fig.1. An application of the electric field $E_{d c}=250 \mathrm{~V} / \mathrm{m}$ decreases the amplitude of the quantum oscillations significantly and at strong magnetic fields the conductance of the structure approaches values that are very close to zero. Shown in Fig. 2, further increase of the dc electric field produces additional peaks in the dependence $g_{12}(B)$, which are labeled by arrows. As shown recently, these maximums result from Zener tunneling between Landau levels, which is induced by applied electric field $E_{d c}{ }^{5}$. Positions of the maximums obey the following relation: $\gamma R_{c} e E_{d c}=l \hbar \omega_{c}$, shown in the insert to Fig.2.

Figure 3a presents dependencies of $g_{12}\left(E_{d c}\right)$ for different magnetic fields as labeled and the temperature $T=1.6 \mathrm{~K}$. At magnetic field $B=0.261 \mathrm{~T}$ the initial drop of the differential conductance with the $E_{d c}$ is due to the intra-level quantal heating ${ }^{7,10}$. The increase of the differential conductance at higher electric field is related to inter-level electron transitions ${ }^{1,37}$. In Fig.3(a) the maximum marked by the arrow corresponds to Zener tunneling between Landau levels at $l=1$. At higher magnetic field $B=0.847 \mathrm{~T}$ the differential conductance demonstrates similar behavior at small electric fields but at higher dc biases the conductance retains values near zero $g_{12} \approx 0$ in a broad range of the electric fields $E_{d c}$. This is the Zero Differential Conductance State (ZDCS). Fig.4(a) reveals that the transition into the ZDC state is associated with one or few sharp "spikes" of the differential conductance into the region with negative values. As shown in the figure the state with $g_{12}=0$ does not occur at $T=4.2 \mathrm{~K}$.

Figure 3(b) presents $V-I$ dependencies of the 2D Corbino disc at temperature $T=1.6 \mathrm{~K}$ for two different magnetic fields as labeled. The figure shows that when the $2 \mathrm{D}$ electron systems enters the state with zero differential conductance, the electric current $I_{d c}$ saturates and becomes independent of the electric field $E_{d c}$. A comparison between the dependencies $g_{12}\left(E_{d c}\right)$ and $I_{d c}\left(V_{d c}\right)$ taken at temperature $\mathrm{T}=1.6 \mathrm{~K}$ and magnetic field $B=0.847 \mathrm{~T}$ indicates that the electric current $I_{d c}$ reaches a saturation value $I_{s}$ at electric field $E_{d c}>E_{t h}$.

Similar to the case of the Hall bar geometry ${ }^{6}$ we consider that in the studied Corbino discs, the $g_{12}=0$ state occurs due to a local instability of the electric field $E_{d c}{ }^{11}$. The dominant nonlinear mechanism, leading to the instability, is a peculiar Joule heating (quantal heating), which occurs in systems with a discrete spectrum ${ }^{7,10}$. The instability develops at the conditions of a negative differ- 

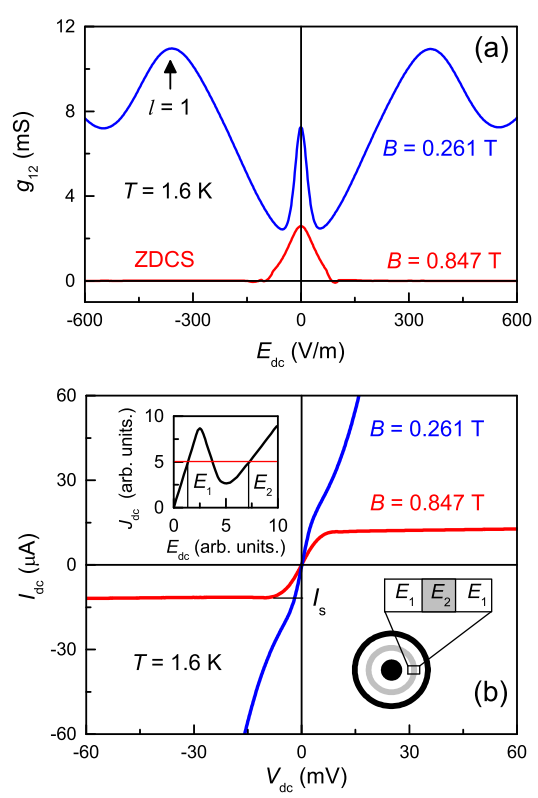

FIG. 3: (a) Dependence of differential conductance $g_{12}$ on dc electric field $E_{d c}$ at different magnetic fields as labeled. Arrow indicates maximum corresponding to Zener transition at $l=1$. $\mathrm{T}=1.6 \mathrm{~K}$; (b) Dependence of electric current $I_{d c}$ on dc voltage $V_{d c}$ at temperature $\mathrm{T}=1.6 \mathrm{~K}$ in different magnetic fields as labeled. Placed in the upper left corner insert shows suggested N-shaped dependence $J_{d c}\left(E_{d c}\right)$ indicating two electric fields $E_{1}$ and $E_{2}$ corresponding to the same value $J_{d c}$. Placed in the lower right corner insert shows possible distribution of the electric field corresponding to the electron state with zero differential conductance in a 2D Corbino disc.

ential conductivity corresponding to the negative slope of the N-shaped V-I dependence shown in the insert to Fig.3(b). Shown in Fig.4(a) regions with the negative differential resistance further supports this interpretation. In the case of the N-shaped V-I dependence, a spatially uniform distribution of the electric field is not stable and typically should evolve into a structure containing domains of a weak $E_{1}$ and a strong $E_{2}$ as shown in the insert to Fig.3(b) ${ }^{39}$. At these conditions both moving and static domains may occur. In the first case in a conductor with a fixed voltage applied there are oscillations of the electric current. This is known as Gunn effect ${ }^{40}$. In the case of static domains the constant electric current saturates with the applied voltage ${ }^{41}$. There is a similarity between nonlinear transport in Gunn diodes ${ }^{40}$ and in the $2 \mathrm{D}$ electron systems presented in this paper. We note however that despite the similarity the nonlinear mechanisms leading to the local instability of the electric field $E_{d c}$ are different in these two systems.

The presented nonlinear response of Corbino discs is obtained in the regime where the edge states and/or skipping orbits are localized near the contacts and do not participate in the electron transport through the systems. It is important to compare the obtained results with the nonlinear response of Hall bar samples, where the electron transport near the edge may provide signif-
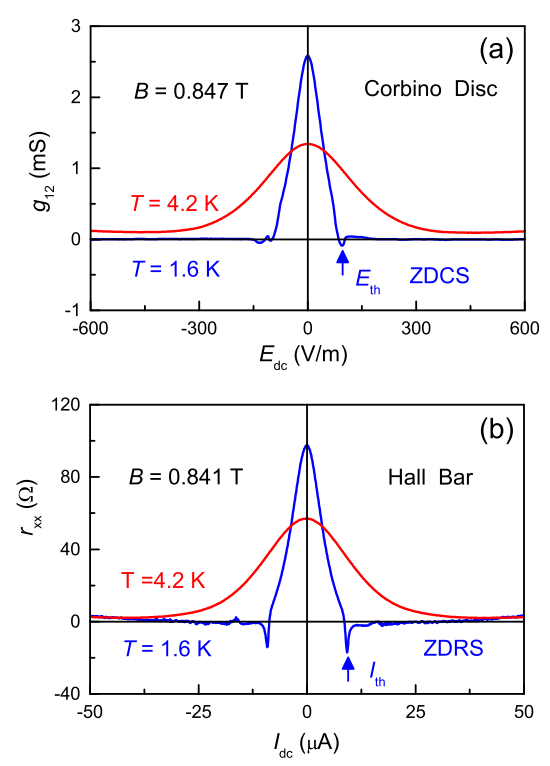

FIG. 4: Color online. (a) Dependence of differential conductance $g_{12}$ on electric field $E_{d c}$ in magnetic field $\mathrm{B}=0.847 \mathrm{~T}$ at different temperatures as labeled. (b) Dependence of the differential resistance $r_{x x}$ on dc bias $I_{d c}$ in Hall bar sample fabricated from the same quantum well as in Fig.4(a). The dependence is taken at magnetic field $\mathrm{B}=0.841 \mathrm{~T}$ at different temperatures as labeled.

icant contributions ${ }^{15}$. Below we compare the threshold electric field $E_{t h}=96 \mathrm{~V} / \mathrm{m}$ corresponding to the transition into the state with zero differential conductance shown in Fig.4a with the Hall electric field corresponding to the transition into the state with zero differential resistance (ZDRS) in a Hall bar sample fabricated from the same quantum well. Figure 4(b) presents the dependence of the differential resistance of the Hall bar sample on the applied dc bias $I_{d c}$ taken at the same experimental conditions. The transition to the ZDR state occurs at Hall electric field $E_{t h}^{H}=118 \mathrm{~V} / \mathrm{m}$, corresponding to the threshold dc bias $I_{d c}=9.3 \mu \mathrm{A}$. The comparison demonstrates quite similar values of the electric fields, at which both ZDRS and ZDCS transitions occur. Furthermore we note that samples with comparable physical parameters demonstrate comparable threshold fields. In particular shown in Fig.2a of ref. $\left.{ }^{6}\right)$ the threshold electric current $I_{t h}=6.7 \mu \mathrm{A}$ corresponds to the ZDRS transition obtained at $\mathrm{B}=0.784 \mathrm{~T}, \mathrm{~T}=1.94 \mathrm{~K}$ on sample $\mathrm{N} 1$ with electron density $n=8.2 \cdot 10^{15} \mathrm{~m}^{-2}$ and mobility $\mu=85 \mathrm{~m}^{2} / \mathrm{Vs}$. Taking into account that the Hall resistance of the sample $\mathrm{N} 1$ at $\mathrm{B}=0.784 \mathrm{~T}$ is $R_{H}=B / n e=597 \mathrm{Ohm}$, one can evaluate the Hall electric field $E_{H}$ corresponding to the current $I_{t h}: E_{t h}^{H}=R_{H} \cdot I_{t h} / W=80 \mathrm{~V} / \mathrm{m}$, where $W=50$ $\mu \mathrm{m}$ is the width of the sample N1. The sample demonstrates similar value of the threshold electric field. Thus in the studied Hall bar samples the edge states and/or skipping orbits do not provide a considerable contribution to the nonlinear response and, thus, the accepted model of the nonlinearity ${ }^{7,10}$ holds for these systems. 
In summary the paper presents experimental study of the effect of dc electric field on the conductance of Corbino discs of highly mobile two dimensional electrons placed in crossed electric and quantizing magnetic fields. Experimental data shows that at low temperature the differential conductance of the Corbino discs reaches zero value in a broad range of applied dc voltages. It indicates the presence of the zero differential conductance state in which the electric current does not depend on the voltage. The results are in accord with the data obtained in the Hall bar geometry indicating that the nonlinearity leading to the ZDC and ZDR states occurs inside 2D electron systems. It provides significant support for the model of the local nonlinearity based on the quantal Joule heating in systems with discrete or modulated spectrum. Finally both the zero differential conductance and zero differential resistance states are observed in systems with a modest electron mobility broadening significantly the class of electron systems in which the quantal heating is essential.

The work was supported by RFBR (project 1102-00925) and National Science Foundation (DMR 1104503).
* Novosibirsk State Technical University, 630092 Novosibirsk, Russia; Rzhanov Institute of Semiconductor Physics, Siberian Branch, Russian Academy of Science, 630090 Novosibirsk, Russia

$\dagger$ Graduate Center, City University of New York

¥ Corresponding author: vitkalov@sci.ccny.cuny.edu

1 C. L.Yang, J. Zhang, and R. R. Du, J. A. Simmons and J. L.Reno, Phys. Rev. Lett. 89, 076801 (2002).

2 A. A. Bykov, Jing-qiao Zhang, Sergey Vitkalov, A. K. Kalagin, and A. K. Bakarov Phys. Rev. B 72, 245307 (2005).

3 A. A. Bykov, JETP Lett. 88, 394 (2008).

4 Yanhua Dai, Z. Q. Yuan, C. L. Yang, R. R. Du, M. J. Manfra, L. N. Pfeiffer, and K. W. West, Phys. Rev. B 80, 041310 (2009)

5 A. A. Bykov, D. V. Dmitriev, I. V. Marchishin, S. Byrnes, and S. A. Vitkalov, Appl. Phys. Lett. 100, 251602 (2012).

6 A. A. Bykov, Jing-qiao Zhang, Sergey Vitkalov, A. K. Kalagin, and A. K. Bakarov, Phys. Rev. Lett. 99, 116801 (2007).

7 I. A. Dmitriev, M. G. Vavilov, I. L. Aleiner, A. D. Mirlin, and D. G. Polyakov, Phys. Rev. B 71, 115316 (2005).

8 Jing-qiao Zhang, Sergey Vitkalov, A. A. Bykov, A. K. Kalagin, and A. K. Bakarov, Phys. Rev. B 75, 081305 (R) (2007).

9 N. Romero Kalmanovitz, A. A. Bykov, Sergey Vitkalov, and A. I. Toropov, Phys. Rev. B 78, 085306 (2008).

10 Jing Qiao Zhang, Sergey Vitkalov, and A. A. Bykov, Phys. Rev. B 80, 045310 (2009).

11 A. V. Andreev, I. L. Aleiner, and A. J. Millis, Phys. Rev. Lett. 91, 056803 (2003).

12 R. G. Mani, J. H. Smet, K. von Klitzing, V. Narayanamurti, W. B. Johnson et al., Nature 420, 646 (2002).

13 M. A. Zudov, R. R. Du, L. N. Pfeiffer, and K. W. West, Phys. Rev. Lett. ]bf 90, 046807 (2003).

14 A. T. Hatke, M. A. Zudov, J. D. Watson, and M. J. Manfra, Phys. Rev. B 85, 121306 (2012)

15 A.D. Chepelianskii, J. Laidet, I. Farrer, D.A. Ritchie, K. Kono, H. Bouchiat, unpublished arXiv:1212.2026

16 R. E. Prange and T. W. Nee, Phys. Rev. B 168779 (1968).

17 M.S. Khaikin, Adv.Phys. 181 (1969).

18 V. S. Tsoi JETP Lett 19, 70 (1974).

19 H. van Houten, C.W.J. Beenakker, J.G. Willliamson, M.E. Brockaart, P.H.M. van Loosdrecht, B.J. van Wees, J.E. Mooij, C.T. Foxon and J.J. Harris, Phys. Rev. B 39, 8556
(1989)

20 B. I. Halperin, Phys. Rev. B 25, 2185 (1982).

21 M. Buttiker, Phys. Rev. B 38, 9375 (1988).

22 P.L. McEuen, A. Szafer, C.A. Richter, B.W. Alphenaar, J.K. Jain, A.D. Stone, R.G. Wheeler and R.N. Sacks, Phys. Rev. Lett. 64, 2062 (1990).

23 A.D. Chepelianskii and D.L. Shepelyansky, Phys. Rev. B 80, 241308(R), (2009)

${ }^{24}$ W. Zhang, M. A. Zudov, L. N. Pfeiffer, and K. W. West, Phys. Rev. Lett. 100, 036805 (2008).

25 A. A. Bykov, JETP Lett. 88, 64 (2008).

26 A. T. Hatke, M. A. Zudov, L. N. Pfeiffer, and K. W. West, Phys. Rev. B 79, 161308 (2009).

27 A. A. Bykov, E. G. Mozulev, and S. A. Vitkalov, JETP Lett. 92, 475 (2010).

28 A. T. Hatke, H.-S. Chiang, M. A. Zudov, L. N. Pfeiffer, and K. W. West, Phys. Rev. B 82, 041304 (2010).

29 Zhenbing Tan, Changling Tan, Li Ma, G. T. Liu, L. Lu, and C. L. Yang, Phys. Rev. B 84, 115429 (2011).

30 A. V. Goran, A. K. Kalagin, and A. A. Bykov, JETP Lett. 94, 535 (2011).

31 S. Wiedmann, G. M. Gusev, O. E. Raichev, A. K. Bakarov, and J. C. Portal, Phys. Rev. B 84, 165303 (2011).

32 Scott Dietrich, Sean Byrnes, Sergey Vitkalov, D. V. Dmitriev, and A. A. Bykov, Phys. Rev. B 85, 155307 (2012).

33 A. T. Hatke, M. A. Zudov, L. N. Pfeiffer, and K. W. West, Phys. Rev. B 86, 081307(R) (2012).

34 Scott Dietrich, Sean Byrnes, Sergey Vitkalov, A. V. Goran, and A. A. Bykov, Phys. Rev. B 86, 075471 (2012).

35 S. A. Studenikin, G. Granger, A. Kam, A. S. Sachrajda, Z. R. Wasilewski, and P. J. Poole, Phys. Rev. B 86, 115309 (2012).

36 K. J. Friedland, R. Hey, H. Kostial, R. Klann, and K. Ploog, Phys. Rev. Lett. 77, 4616 (1996).

37 M. G. Vavilov, I. L. Aleiner, and L. I. Glazman, Phys. Rev. B 76, 115331 (2007).

38 C. Faugeras, D. K. Maude, G. Martinez, L. B. Rigal, C. Proust, K. J. Friedland, R. Hey, and K. H. Ploog, Phys. Rev. B 69, 073405 (2004).

39 B. K. Ridley, Proc. Phys. Soc. 82, 954 (1963).

40 J. B. Gunn, Solid State Commun. 1, 88 (1963).

41 M. E. Levinshtein, and M. S. Shur, Sov. Phys. Semicond. 3, 915 (1970). 